\title{
Análise da Mortalidade Evitável de Mulheres em Idade Reprodutiva
}

Analysis of Avoidable Mortality among Women in Reproductive Age

Mary Angela Parpinelli, Aníbal Faúndes, José Guilherme Cecatti, Belmiro Gonçalves Pereira Renato Passini Júnior, Eliana Amaral

\begin{abstract}
RESUMO
Objetivos: avaliar a mortalidade evitável de mulheres em idade reprodutiva, residentes em Campinas, SP, comparando os qüinqüênios de 1985-89 com 1990-94.

Métodos: foram estudadas 3.086 declarações de óbito de mulheres de 10 a 49 anos, que foi o número total de mortes no periodo de janeiro de 1985 a dezembro de 1994. Foram aplicados os critérios de evitabilidade, segundo medidas preventivas, medidas de diagnóstico e tratamento precoces, medidas de saneamento e medidas mistas. Foram ainda classificadas de causa dificilmente evitável, as mal definidas e as por outras causas. Procedeu-se ao cálculo dos coeficientes de mortalidade especificos para cada periodo de cinco anos e da razão entre estes coeficientes.

Resultados: houve aumento na taxa de mortalidade evitável em 20\% na comparação entre os dois periodos. As maiores falhas ocorreram no grupo de causas evitáveis por medidas preventivas e de saneamento. O maior aumento em causa de morte por medidas preventivas foi resultado da AIDS. Dentre as mortes evitáveis por medidas mistas, deve-se ressaltar o aumento de 50\% tanto para mortalidade materna por aborto, quanto nas mortes violentas, principalmente homicídios.

Conclusões: houve aumento na proporção de mortes por causas evitáveis. Medidas para prevenção da AIDS, do aborto provocado e para redução das mortes violentas, principalmente homicidios, deveriam ser prioridades político-sociais no país.
\end{abstract}

PALAVRAS-CHAVE: AIDS, Saneamento básico. Mortalidade feminina. Saúde reprodutiva.

\section{Introdução}

O conceito de mortalidade prematura foi introduzido por Dempsey ${ }^{1}$ ao analisar a mortalidade secundária à tuberculose ${ }^{1}$. $O$ princípio fundamenta-se em quantificar a perda, segundo aspectos sociais, políticos, intelectuais e econômicos, além dos aspectos pessoais e familiares acarretados pela morte que ocorre prematuramente, ou seja, prévia à idade definida pela ex-

Disciplina de Obstetrícia, Faculdade de Ciências Médicas

Departamento de Tocoginecologia

Universidade Estadual de Campinas

Correspondência:

Mary Angela Parpinelli

Caixa Postal 6030

Rua: Alexander Fleming, 101

13092-340 - Campinas - SP

Fone: (19) 788-7910 - Fax (19) 7889304 pectativa de vida ao nascimento.

Diferentes métodos têm sido utilizados para medir a mortalidade prematura. Um deles é quantificá-la a partir do indicador "Anos Potenciais de Vida Perdidos", técnica que se fundamenta no cálculo matemático a partir do limite inferior de idade pré-estabelecido (nascimento, infância, adolescência ou outro) e como limite superior, em geral, a idade correspondente à expectativa de vida ao nascimento ${ }^{2}$.

A mortalidade prematura também pode ser avaliada segundo a identificação das causas de morte ajustadas por idade e sexo, adaptadas à possibilidade de prevenção do óbito, a partir de conhecimentos e tecnologias disponiveis nos diferentes países de aplicação. Este método de análise foi descrito na Europa e definiu-se como estudo da mortalidade evitável a partir de uma 
listagem de doenças consideradas como de causas evitáveis de morte para os padrões locais ${ }^{3}$.

O conceito de morte evitável ganhou força, principalmente nos países europeus, e passou a ser considerado como indicador da qualidade dos cuidados à saúde, durante as últimas déca$\operatorname{das}^{4-6}$. O método de mortalidade evitável pode ser aplicado para estabelecer comparação dos cuidados de saúde entre diferentes países ${ }^{7}$, distintos serviços de saúde, em relação a distintos períodos de tempo ${ }^{6}$ e entre diferentes classes sócio-econômicas ${ }^{8}$.

Recentemente, Treurniet et al. ${ }^{9}$ propuseram que a comparação da mortalidade evitável entre países ou regiões deveria ser ajustada segundo variações de incidência e gravidade das doenças, dado que seria mais sensivel para avaliar a qualidade de saúde que a taxa bruta de mortalidade.

No continente americano, um dos pioneiros no estudo da mortalidade evitável foi Taucher ${ }^{10}$, em estudo sobre mortalidade, realizado no Chile. A autora não utilizou lista de doenças, mas sim definiu um conjunto de critérios que individualmente compõem a possibilidade de se evitar o óbito. O presente estudo propõese a determinar e avaliar a mortalidade evitável de mulheres em idade reprodutiva, de 10 a 49 anos, residentes na cidade de Campinas, região sudeste do Estado de São Paulo, Brasil, comparando os qüinqüênios 1985-89 e 1990-94.

\section{Métodos}

Foram estudadas 3.086 declarações de óbito (DO) de mulheres de 10 a 49 anos, residentes no município de Campinas (SP), número que representa o total de óbitos femininos ocorridos no período de primeiro de janeiro de 1985 a 31 de dezembro de 1994, para a faixa etária específica. Detalhes relativos ao método utilizado no estudo encontram-se descritos em outro $\operatorname{artigo~}^{11}$.

Procedeu-se ao cálculo dos coeficientes de mortalidade específicos e aplicando-se os critérios de evitabilidade descritos por Taucher ${ }^{10}$. Após avaliação final de cada óbito, aplicaram-se os critérios de evitabilidade da morte, que classificam as causas de morte evitáveis em quatro categorias: a) evitáveis por medidas preventivas (vacinação, doenças sexualmente transmissíveis); b) evitáveis por diagnóstico e tratamento precoces; c) evitáveis por medidas de saneamento básico; d) evitáveis por aplicação de conjunto de medidas (sociais, diferentes níveis de atenção mé- dica).

Tais critérios classificam ainda outras causas como as mortes dificilmente evitáveis (segundo nível de tecnologia disponível para o momento e/ou pelo tipo de população). Nesta categoria encontra-se a maior parte das neoplasias malignas, doenças cardiovasculares e neurológicas. A estas se acrescentam as mortes por causas mal definidas e outras causas de morte.

O presente estudo utiliza tal classificação, introduzindo as seguintes modificações:

1. Doença causada pelo vírus da imunodeficiência humana (HIV) - CID $10^{\mathrm{a}}$ revisão $^{12}$ (B20B24), classificada como causa evitável por medidas preventivas.

2. Neoplasia maligna de mama (C50), retirada do critério de morte evitável por diagnóstico e tratamento precoces e introduzida no critério de morte dificilmente evitável.

3. Neoplasia maligna da placenta (C58), neoplasia maligna de vulva (C51) e neoplasia maligna de vagina (C52), categorizadas como mortes evitáveis por diagnóstico e tratamento precoces.

4. Septicemia (A40-A41); neoplasias malignas não especificadas (C76-C80); distúrbios da coagulação (D65); doenças cardíacas mal definidas, como pericardite aguda não-especificada (I30), tamponamento cardiaco (I31), parada cardiaca não-especificada (I46), insuficiência cardíaca não-especificada (I50); edema agudo do pulmão (J80-J84), pneumotórax (J93), derrame pleural (J90), classificadas como mortes por causas mal definidas.

Para análise da evitabilidade, foram alocadas as causas de óbito segundo os critérios utilizados e calculados os coeficientes de mortalidade específicos para dois períodos (198589 e 1990-94), estabelecendo-se então a razão entre os coeficientes.

\section{Resultados}

A Tabela 1 mostra que o número de óbitos, bem como os coeficientes de mortalidade específicos por causas possivelmente evitáveis, aumentaram em torno de $20 \%$ entre o primeiro e o segundo período analisado, com razão entre os coeficientes de 1,2. Para as causas de morte classificadas como dificilmente evitáveis, causas mal definidas e por outras causas houve pequenas variações, com razões entre as taxas oscilando entre $1,1,0,9$ e 0,8 , respectivamente.

Dentre o conjunto de medidas que poderi- 
am ser aplicadas para se evitarem estes óbitos, as medidas preventivas foram as mais deficitárias, com razão entre os coeficientes de 4,7 com- parando-se os dois períodos. Esta diferença explica-se, basicamente, pelo aumento na mortalidade por AIDS (Tabela 2).

Tabela 1 - Coeficientes de mortalidade específicos e razão entre os coeficientes das mortes de mulheres de 10 a 49 anos, residentes em Campinas, nos períodos de 1985-89 e 1990-94, segundo evitabilidade.

\begin{tabular}{lrrrrr}
\hline Critérios & $\mathbf{n}$ & $\mathbf{1 9 8 5 - 8 9}$ & \multicolumn{1}{c}{ 1990-94 } & \\
\hline Mortes evitáveis & 583 & 45,2 & 768 & 53,3 & Razão** $^{*}$ \\
$\quad$ Medidas preventivas & 25 & 1,9 & 129 & 8,9 & 1,2 \\
$\quad$ Diagnóstico/tratamento precoces & 78 & 6,0 & 85 & 5,9 & 4,7 \\
$\quad$ Medidas de saneamento & 21 & 1,6 & 31 & 2,1 & 1,0 \\
$\quad$ Medidas mistas & 459 & 35,6 & 523 & 36,3 & 1,3 \\
Mortes dificilmente evitáveis & 431 & 33,4 & 527 & 36,6 & 1,0 \\
Causas mal definidas & 171 & 13,3 & 165 & 11,4 & 1,1 \\
Mortes por outras causas & 229 & 17,8 & 212 & 14,7 & 0,9 \\
Total & 1414 & 109,7 & 1672 & 116,0 & 0,8 \\
\hline
\end{tabular}

CME (coeficiente de mortalidade especifico para os periodos) por 100.000 mulheres de $10-49$ anos

${ }^{* *}$ Razão entre CME 1990-94/CME 1985/89

Tabela 2-Coeficientes de mortalidade específicos e razão entre coeficientes das mortes evitáveis por medidas preventivas, de mulheres de 10 a 49 anos, residentes em Campinas, nos períodos de $1985-89$ e $1990-94$

\begin{tabular}{lrrrrr}
\hline \multirow{2}{*}{ Causas } & \multicolumn{2}{c}{$1985-89$} & \multicolumn{2}{c}{$1990-94$} & \\
\hline AIDS & n & CME* & n & CME* & Razão** \\
Meningite e outras & 8 & 0,6 & 106 & 7,3 & 12,2 \\
Doenças reumáticas & 11 & 0,5 & 12 & 0,8 & 1,6 \\
Total & 25 & 1,9 & 11 & 0,8 & 1,0 \\
\hline
\end{tabular}

* CME (coeficiente de mortalidade específico para os períodos) por 100.000 mulheres de 10-49 anos

** Razão entre CME 1990-94/CME 1985/89
Não houve variação entre os coeficientes brutos nas mortes evitáveis por medidas de diagnóstico e tratamento precoces entre os dois qüinqüênios. Deve-se ressaltar, entretanto, o aumento nos coeficientes de mortalidade por câncer de pele e doenças renais agudas (Tabela 3). A mortalidade evitável por medidas de saneamento aumentou à razão de 1,3 vezes no segundo período em relação ao primeiro, porém o número de mortes por estas causas foi pequeno (Tabela 4).

Tabela 3 - Coeficientes de mortalidade específicos e razão entre coeficientes das mortes evitáveis por medidas de diagnóstico e tratamento precoces, de mulheres de 10 a 49 anos, residentes em Campinas, nos períodos de $1985-89$ e $1990-94$.

\begin{tabular}{|c|c|c|c|c|c|}
\hline \multirow[b]{2}{*}{ Causas } & \multicolumn{2}{|c|}{$1985-89$} & \multicolumn{2}{|c|}{$1990-94$} & \multirow[b]{2}{*}{ Razão** } \\
\hline & $\mathrm{n}$ & $\mathrm{CME}^{*}$ & n & $\mathrm{CME}^{*}$ & \\
\hline Ca de pele & 4 & 0,3 & 10 & 0,7 & 2,3 \\
\hline Ca de vulva e vagina & 3 & 0,2 & - & - & - \\
\hline Ca de colo uterino & 38 & 2,9 & 33 & 2,3 & 0,8 \\
\hline Ca de útero e não especificado & 6 & 0,5 & 7 & 0,5 & 1,0 \\
\hline Ca de placenta & 1 & 0,07 & 1 & 0,06 & 0,8 \\
\hline Úlcera, apendicite, hérnia, colecistopatia & 23 & 1,8 & 25 & 1,7 & 0,9 \\
\hline Doenças renais agudas & 3 & 0,2 & 9 & 0,6 & 3,0 \\
\hline Total & 78 & 6,0 & 85 & 5,9 & 1,0 \\
\hline
\end{tabular}

${ }^{*} \mathrm{CME}$ (coeficiente de mortalidade específico para os períodos) por 100.000 mulheres de $10-49$ anos

** Razão entre CME 1990-94/CME 1985/89 
Tabela 4 - Coeficientes de mortalidade específicos e razão entre coeficientes das mortes evitáveis por medidas de saneamento, de mulheres de $10 \mathrm{a} 49$ anos, residentes em Campinas, nos períodos de $1985-89$ e 1990-94.

\begin{tabular}{|c|c|c|c|c|c|}
\hline \multirow[b]{2}{*}{ Causas } & \multicolumn{2}{|c|}{ 1985-89 } & \multicolumn{2}{|c|}{$1990-94$} & \multirow[b]{2}{*}{ Razão** } \\
\hline & $\mathbf{n}$ & $\mathrm{CME}^{*}$ & $\mathrm{n}$ & $\mathrm{CME}^{*}$ & \\
\hline Diarréia, gastroenterites & 2 & 0,1 & 3 & 0,2 & 2,0 \\
\hline Tripanosomíase & 16 & 1,2 & 20 & 1,4 & 1,2 \\
\hline Helmintíases & 1 & 0,07 & 3 & 0,2 & 2,8 \\
\hline Toxoplasmose/protozoários & 1 & 0,07 & 2 & 0,1 & 1,4 \\
\hline Outras & 1 & 0,07 & 3 & 0,2 & 2,8 \\
\hline Total & 21 & 1,6 & 31 & 2,1 & 1,3 \\
\hline
\end{tabular}

${ }^{*} \mathrm{CME}$ (coeficiente de mortalidade específico para os períodos) por 100.000 mulheres de 10-49 anos

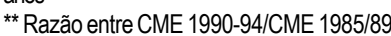

Dentre as mortes evitáveis com medidas definidas como mistas, encontraram-se os óbitos maternos por aborto, que apresentaram ascensão de $50 \%$ entre o primeiro e o segundo período, mas houve apenas 5 e 7 casos em cada periodo, respectivamente. Aumentaram também as mortes por acidentes e violência, particularmente as por homicídios (Tabela 5).

Tabela 5- Coeficientes de mortalidade específicos e razão entre coeficientes das mortes evitáveis por medidas mistas, de mulheres de 10 a 49 anos, residentes em Campinas, nos períodos de 1985-89 e 199094.

\begin{tabular}{lrrrrr}
\hline Causas & \multicolumn{2}{c}{$\mathbf{1 9 8 5 - 8 9}$} & \multicolumn{1}{c}{$\mathbf{1 9 9 0 - 9 4}$} & \\
\hline Pneumonia & $\mathbf{n}$ & $\mathbf{C M E}$ & $\mathbf{n}$ & $\mathbf{C M E}$ & Razão \\
Doenças do fígado & 81 & 6,3 & 82 & 5,7 & 0,9 \\
Tuberculose (s/ref. AIDS) & 37 & 2,9 & 46 & 3,2 & 1,1 \\
Hepatite viral & 12 & 0,9 & 8 & 0,5 & 0,5 \\
Anemia/desnutrição & 5 & 0,4 & 10 & 0,7 & 1,7 \\
\# MM por aborto & 7 & 0,5 & 2 & 0,1 & 0,2 \\
\# MM direta & 5 & 5,2 & 7 & 8,0 & 1,5 \\
\# MM indireta & 28 & 29,2 & 25 & 28,4 & 1,0 \\
Acidentes de trânsito & 11 & 11,5 & 6 & 6,8 & 0,6 \\
Homicídios & 123 & 9,5 & 115 & 8,0 & 0,8 \\
Outros acidentes, não-especificado & 32 & 2,5 & 55 & 3,8 & 1,5 \\
Total & 118 & 9,1 & 167 & 11,6 & 1,3 \\
\hline
\end{tabular}

${ }^{*} \mathrm{CME}$ (coeficiente de mortalidade específico para os períodos) por 100.000 mulheres de 10-49 anos

** Razão entre CME 1990-94/CME 1985/89

\# NV (Nascidos Vivos) 1985-89=95.894/1990-94=87.930

\section{Discussão}

Estes resultados contribuem para mostrar que cerca da metade das mortes entre as mulheres em idade reprodutiva é evitável com medidas de complexidade variada, mas cujo custo é, sem dúvida, menor que a perda pessoal, social e econômica. Analisando com mais detalhe, observa-se que o coeficiente de mortalidade específico passou de 45,2 para 53,3 mortes por 100.000 mulheres de 10-49 anos nos períodos de 1985-89 e 1990-94, respectivamente.

Os critérios inicialmente estabelecidos por Taucher ${ }^{10}$ foram adaptados para algumas situações. O câncer de mama foi excluído da categoria de morte evitável por medidas de diagnóstico e tratamento precoces, sendo classificado como de morte dificilmente evitável. Isto se deve à maior morbidade do câncer de mama em mulheres jovens e às dificuldades nos métodos de triagem. A mamografia (a partir dos 40 anos) é o mais importante método disponivel para diagnóstico precoce do câncer de mama. Associada ao exame clinico e ao auto-exame das mamas, é capaz de detectar 95\% dos casos de câncer de mama, o que não significa que seja capaz de evitar a morte por esta causa. Além disso, estima-se uma taxa de falso negativo de até $20 \%$ com a mamografia ${ }^{13}$.

A CID- $10^{12}$ introduziu a doença causada pelo vírus da imunodeficiência humana (HIV) no capítulo I (doenças infecciosas e parasitárias A00B99), retirando-a do capítulo III, CID $9^{14}$ (doenças imunológicas). $\mathrm{Na}$ época em que foram defi- 
nidos os critérios, ainda não se conhecia a AIDS e, dessa forma, a doença não consta das definições propostas por Taucher ${ }^{10}$. Decidimos incluíla no capítulo de morte evitável segundo medidas preventivas, uma vez que fazem parte deste capítulo as demais doenças de transmissão preferencialmente sexual, além do que, ainda nos dias atuais, a melhor maneira de se evitar a morte por AIDS e suas complicações é evitando adquirir a doença.

A correlação entre a infecção pelo HIV e fatores comportamentais, como sexo desprotegido, uso de drogas injetáveis, entre outros, reforça a inclusão da AIDS como morte evitável por medidas de prevenção. McGinnis \& Foege ${ }^{15}$ avaliaram a mortalidade, nos Estados Unidos, com a perspectiva de correlacionar fatores comportamentais às causas de morte e mostraram, por exemplo, que em 1990 aproximadamente 9.000 mortes por AIDS foram secundárias em primeira instância ao uso de drogas endovenosas. Mostraram ainda que 30.000 mortes, também em 1990, podem ser atribuídas ao sexo desprotegido, sendo 5.000 por aumento nas taxas de mortalidade infantil secundário à gravidez indesejada, 4.000 por câncer de colo uterino e 21.000 por HIV de transmissão sexual. Realizaram ainda várias outras associações de atitudes comportamentais e hábitos com outras causas de morte.

A taxa de mortalidade com possibilidades de ser evitada aumentou, quando se comparou o período 1985-89 com o período 1990-94. Este aumento na mortalidade evitável deveu-se, em grande parte, aos óbitos secundários a falhas nas medidas de prevenção e particularmente à AIDS, que representaram um número muito pequeno no primeiro período (8 mortes), quando comparado ao segundo (106 mortes). Os primeiros casos de óbitos por AIDS, nesta população, foram descritos a partir de 1987, o que torna desigual a divisão dos períodos (1985-89 e 1990-94) para os casos de morte por esta causa. Apesar disso, a ascensão da mortalidade, em todas as faixas etárias, foi evidente a partir de 1990.

A razão entre os coeficientes de mortalidade, para os dois periodos, não diferiu no critério estabelecido como morte evitável por medidas de diagnóstico e tratamento precoces, o que pode ser interpretado como provável boa assistência médica dispensada a este grupo da população. Ressalva deve ser feita ao aumento nas mortes secundárias a carcinoma de pele e por doenças renais agudas.

Chama a atenção também o aumento nas taxas de mortalidade evitável por medidas de saneamento, com um aumento de 1,3 vezes em relação ao segundo período. Este aumento na mortalidade por questões relacionadas ao saneamento pode ser interpretado pelo empobrecimento de segmentos da população migrante para a cidade, como grupos de pessoas que se autodenominam "sem teto", e que têm promovido loteamentos clandestinos, sem infra-estrutura básica, localizados principalmente na periferia da cidade, na última década.

O critério definido como morte evitável por medidas mistas é extenso e inclui um conjunto de atitudes médico-sociais e politicas. A mortalidade por causas externas, componente deste grupo, aumentou em 10\% quando comparados os períodos de 1985-89 e 1990-94. Na análise pormenorizada das causas externas de mortalidade, os coeficientes específicos por acidentes de trânsito sofreram queda de $20 \%$ e a mortalidade por homicídios apresentou franca expansão, com razão entre os coeficientes de 1,5. Estes resultados confirmam o crescimento da mortalidade por causas externas, principalmente por homicídios, nas grandes cidades do Brasil, avaliada para o período de 1977 a $1994{ }^{16}$. Ainda neste grupo de causas, o aumento de $50 \%$ na mortalidade materna secundária às complicações do aborto, apesar do pequeno número de casos, sugere a necessidade de medidas político-sociais para controle destas mortes evitáveis.

Os óbitos devidos a causas mal definidas apresentaram coeficientes de mortalidade superiores a 10 mortes / 100.000 mulheres de 10-49 anos, em ambos os periodos estudados. Entretanto, é importante ressaltar que foram incluídas nesta categoria algumas doenças que, embora apresentem uma codificação para causa de morte, foram consideradas inespecíficas, como septicemia, edema agudo de pulmão, neoplasia maligna de localização não especificada e outras. Os elevados coeficientes para esta categoria podem ser interpretados como devidos à má qualidade do preenchimento das declarações de óbito ou ainda pelas más condições de assistência médica.

As outras causas de morte representadas pela doenças hematológicas, diabete, pulmonares crônicas, colagenoses, renais e algumas cardiovasculares, como as doenças hipertensivas, apresentaram razão inferior a um, quando comparados os dois periodos, o que significa um aparente decréscimo de ocorrência.

Em termos de evitabilidade, o que mais chama a atenção é o elevado número de mortes violentas, seja por acidentes de trânsito, outros acidentes ou homicídios. Estas foram as causas de morte de 273 mulheres no período de 198589 e de 337 em 1990-94, correspondendo à taxa, respectivamente, de 21,2 e 23,4 mortes por 100.000 mulheres de 10-49 anos em cada perio- 
do analisado. Apesar de representar um número de mortes mais de oito vezes superior às mortes maternas, pouco se discute este problema, nem existem campanhas nacionais sistematicamente organizadas para preveni-las.

Tal situação é também diferente da observada quanto a AIDS, que é objeto de amplas campanhas de informação e prevenção da doença. Existe pouca consciência, entretanto, do assustador crescimento da infecção pelo HIV como causa de morte de mulheres jovens, que passou de 8 no periodo de $1985-89$ para 106 casos nos cinco anos seguintes. Se a curva ascendente se mantiver, é possivel que, em poucos anos, as mortes por esta única doença passem a ser em maior número que aquelas devidas a causas listadas em qualquer capítulo da CID.

Espera-se que os dados apresentados neste estudo não só contribuam a criar uma nova consciência sobre a dimensão pouco divulgada destas causas de óbito, mas que também estimulem a continuidade da monitorização do que acontece com a mortalidade feminina, além do interesse particular sobre as mortes maternas.

\section{SUMMARY}

Purpose: to evaluate the avoidable mortality among women in reproductive age, living in Campinas, SP, comparing two five-year periods: $1985-89$ and 1990-94.

Methods: death certificates of 3.086 women aged 10 to 49 years were studied, representing the total number of deaths during the period from January 1985 through December 1994. The criteria for avoidance were applied to these deaths using preventive, sanitary, early diagnosis and treatment, and mixed measures. The deaths were also classified as: with hardly avoidable causes, not welldefined causes and other causes. The specific mortality coefficient for each period of five years and the ratio between these coefficients were calculated.

Results: there was a $20 \%$ increase in the avoidable mortality rate from the first to the second period. The main failure was observed among the group of avoidable causes by preventive and sanitary measures. The main increase in death causes by preventive measures resulted from AIDS. Among the causes of death avoidable by mixed measures, the increase of 50\% in maternal mortality caused by abortion, as well as causes due to violence specially homicides, are emphasized.

Conclusion: there was an increase in the proportion of avoidable death causes. Measures to prevent AIDS, abortion and to reduce violent deaths, specially homicides, should be political and social priorities in our Country.

KEY WORDS: AIDS. Avoidable mortality. Women mortality. Reproductive health.

\section{Referências}

1. Dempsey M. Decline in tuberculosis: the death rate fails to tell the entire story. Am Rev Tuberc 1947; $56: 157-64$.

2. Romeder JM, McWhinnie JR. Potential years of life lost between ages 1 and 70: an indicator of premature mortality for health planning. Int $\mathrm{J}$ Epidemiol 1977; 6:143-51.

3. Rutstein DD, Berenberg W, Chalmers TC, Child CG $3^{\text {rd }}$, Fishman AP, Perrin EB. Measuring the quality of medical care. A clinical method. N Engl J Med 1976; 294: 582-8.

4. Charlton JR, Hartley RM, Silver R, Holland WW. Geographical variation in mortality from conditions amenable to medical intervention in England and Wales. Lancet 1983; 1:691-6.

5. Holland WW. Avoidable death as a measure of quality. Qual Assur Health Care 1990; 2:227-33.

6. Westerling $\mathrm{R}$. Trends in avoidable mortality in Sweden, 1974-1985. J Epidemiol Community Health 1992; 46:489-93.

7. Gaizauskiene A, Westerling R. A comparison of avoidable mortality in Lithuania and Sweden 1971-1990. Int J Epidemiol 1995; 24:1124-31.

8. Westerling R, Gullberg A, Rosen M. Socioeconomic differences in avoidable mortality in Sweden, 1986-1990. Int J Epidemiol 1996; 25:560-7.

9. Treurniet $\mathrm{HF}$, Looman $\mathrm{CW}$, van der Maas PJ, Mackenbach JP. Variations in avoidable mortality: a reflection of variations in incidence? Int J Epidemiol 1999; 28:225-32.

10.Taucher E. Chile: mortalidad desde 1955 a 1975. Tendências y causas. Santiago de Chile: CELADE; 1978. p.1-83 (Serie A, 162).

11.Faúndes A, Parpinelli MA, Cecatti JG. Mortalidade de mulheres em idade fértil, em Campinas (19851994). Cad Saúde Pública. In press 2000.

12. Organização Mundial da Saúde. Classificação estatística internacional de doenças e problemas relacionados à saúde: CID-10. $2^{\mathrm{a}}$ ed. São Paulo: Centro Colaborador da OMS para a Classificação de Doenças em Português; 1995. v.1.

13. Michigan Department of Public Health. Breast cancer screening and detection in Michigan. Recommendations to reduce mortality. Lansing: Michigan Department of Public Health, Center for Health Promotion; 1990.

14.Organização Mundial da Saúde. Manual da classificação estatística internacional das doenças, lesões e causas de óbito: CID. $9^{a}$ Revisão São Paulo: Centro da OMS para Classificação de Doenças em Português; 1975.

15.McGinnis JM, Foege W. Actual causes of death in the United States. JAMA 1993; 270:2207-12.

16. Mello Jorge MHP, Gawryszewski VP, Latorre MRDO. Accidents and acts of violence in Brazil. I. Analysis of mortality data. Rev Saúde Pública 1997; 31 Supl 4:5-25. 